\title{
Adaptive Controllers and Robustness Analysis for Curve Tracking with Unknown Control Gains
}

\author{
Michael Malisoff
}

\author{
Fumin Zhang
}

\begin{abstract}
We study adaptive control and parameter identification for robotic curve tracking under unknown control gains. We build adaptive controllers that identify the unknown control gains and stabilize equilibria corresponding to a fixed constant distance to the curve and zero bearing. Our strict Lyapunov function method allows us to prove robust performance under actuator errors in terms of integral input-to-state stability under a bound on the disturbance that maintains forward invariance of a class of invariant hexagons. This extends existing curve tracking results to allow controller uncertainty and parameter identification. We demonstrate our work in simulations.
\end{abstract}

Key Words-Curve tracking, robotics, robustness

\section{INTRODUCTION}

Curve tracking methods have proven to be effective for navigating mobile robots in complex environments [5], [15], [17]. For wheeled mobile robots, feedback control laws have been developed that achieve autonomous tracking of obstacle boundaries or a desired smooth path [10]. There were several recent improvements for curve tracking control [11]. These include extensions to the three dimensional case [3] and cooperative control for ocean sensing [16], [18].

Evidence of robustness in real life applications has been reported for farming, obstacle avoidance in corridors, and ocean sampling [4], [16], [19]. Our previous work [7] theoretically justified the robustness by showing that the feedback control laws for two-dimensional curve tracking from [17] provide input-to-state stability (ISS) properties with respect to additive uncertainty on the controller. We proved robust forward invariance of a nested sequence of hexagons $H_{1} \subseteq H_{2} \subseteq H_{3} \subseteq$.. that fill the state space. This produced a sequence of positive constants $\left\{\delta_{i}^{*}\right\}$ such that the following holds for all $i$ : all trajectories of the curve tracking dynamics that start in $H_{i}$ and have uncertainties bounded by $\delta_{i}^{*}$ remain in $H_{i}$ for all positive times. Then we proved ISS of the curve tracking dynamics on each hexagon $H_{i}$, with respect to disturbances bounded by $\delta_{i}^{*}$. This theoretical understanding is important for giving predictable tolerance and safety bounds for the curve tracking control laws. See [12]-[14] for background on ISS.

This paper continues our search for robust controller designs for curve tracking dynamics that respect the constraints

Malisoff is with Department of Mathematics, Louisiana State University, 303 Lockett Hall, Baton Rouge, LA 70803-4918, malisoff@1su.edu.

Zhang is with the School of Electrical and Computer Engineering, Georgia Institute of Technology, 210 Technology Circle, Savannah, GA 31322, fumin@gatech.edu.

The first author was supported by AFOSR Grant FA9550-09-1-0400 and NSF Grant ECCS-1056255. The second author was supported by ONR Grants N00014-08-1-1007 and N00014-09-1-1074, and NSF Grants ECCS0841195, ECCS-0845333 (CAREER), ECCS-1056253, and CNS-0931576. that prevail in real time experimental implementations. We are motivated by our recent deployment of a fleet of marine robots at Grand Isle State Park, Louisiana which surveyed residual crude oil components from the Deepwater Horizon oil spill disaster [2]. In this application, we implemented curve tracking behaviors on two different marine robots with unknown actuator parameters. We implemented the curve tracking control laws from [7], [17] by testing different control gains, and the experimental results will be reported in other publications.

However, the control laws in [7], [17] would be more applicable to real systems if they could be made adaptive to unknown actuation parameters. In this paper, we present an adaptive controller design that can be implemented on the marine robots. We also prove that the adaptive control laws are integral ISS (iISS) with respect to actuation errors. Our adaptive curve tracking result is based on an extension of the barrier Lyapunov function approach from [9] to systems with state constraints, combined with an adaptive version of the robust forward invariant hexagon argument from [7], and includes parameter identification. It differs from the usual adaptive control problem of stabilizing an equilibrium point while keeping the estimate of the unknown parameter bounded, which is more common in the literature. For complete proofs of all results to follow, see [8].

\section{Notation AND DEFINITIONS}

A continuous function $\gamma:[0,+\infty) \rightarrow[0,+\infty)$ is of class $\mathscr{K}$ (written $\gamma \in \mathscr{K}$ ) provided it is strictly increasing and $\gamma(0)=0$; if, in addition, $\gamma(r) \rightarrow+\infty$ as $r \rightarrow+\infty$, then it is of class $\mathscr{K}_{\infty}$ (written $\gamma \in \mathscr{K}_{\infty}$ ). A continuous function $\beta$ : $[0,+\infty) \times[0,+\infty) \rightarrow[0,+\infty)$ is of class $\mathscr{K} \mathscr{L}$ (written $\beta \in$ $\mathscr{K} \mathscr{L}$ ) provided (i) for each fixed $s \geq 0$, the function $\beta(\cdot, s) \in$ $\mathscr{K}$ and (ii) for each fixed $r \geq 0$, the function $\beta(r, \cdot)$ is nonincreasing and $\beta(r, s) \rightarrow 0$ as $s \rightarrow+\infty$. Let $\mathscr{U}$ be any subset of a Euclidean space such that $0 \in \mathscr{U}$, and let $\mathscr{M}_{\mathscr{U}}$ denote the set of all measurable locally essentially bounded functions $\delta:[0,+\infty) \rightarrow \mathscr{U}$. Let $|f|_{\mathscr{S}}$ denote the essential supremum of the restriction of any function $f$ to any subset $\mathscr{S}$ of its domain.

Consider any subset $\mathscr{G}$ of a Euclidean space and any point $\mathscr{E} \in \mathscr{G}$. A continuous function $\mathscr{V}: \mathscr{G} \rightarrow[0,+\infty)$ is positive semi-definite with respect to $\mathscr{E}$ provided $\mathscr{V}(\mathscr{E})=0$; if, in addition, $\mathscr{V}(q)>0$ for all $q \in \mathscr{G} \backslash\{\mathscr{E}\}$, then $\mathscr{V}$ is positive definite with respect to $\mathscr{E}$. A function $\mathscr{V}$ is negative semidefinite (resp., definite) with respect to $\mathscr{E}$ provided $-\mathscr{V}$ is positive semi-definite (resp., definite) with respect to $\mathscr{E}$. Let $|p|_{\mathscr{E}}=|p-\mathscr{E}|$ denote the distance between any point $p \in \mathscr{G}$ 
and the point $\mathscr{E}$ in the usual Euclidean metric. A function $\mathscr{V}$ : $\mathscr{G} \rightarrow[0,+\infty)$ is a modulus with respect to $(\mathscr{E}, \mathscr{G})$ provided it is positive definite with respect to $\mathscr{E}$ and radially unbounded in the following sense: For each constant $M>0$, there is a constant $\delta_{M}>0$ (depending on $M$ ) such that $\mathscr{V}(x) \geq M$ for all $x \in \mathscr{G}$ that satisfy either distance $(x$, boundary $(\mathscr{G})) \leq \delta_{M}$ or $|x|_{\mathscr{E}} \geq 1 / \delta_{M}$. For any subset $\mathscr{S} \subseteq \mathbb{R}^{n}$ and any point $\bar{p} \in \mathbb{R}^{n}$, we set $\mathscr{S}-\bar{p}=\{q-\bar{p}: q \in \mathscr{S}\}$.

Consider a forward complete system $\dot{x}=\mathscr{F}(x, \delta)$ with state space $\mathscr{G}$ and disturbances $\delta \in \mathscr{M}_{\mathscr{U}}$, where $\mathscr{F}: \mathscr{G} \times$ $\mathscr{U} \rightarrow \mathscr{G}$ satisfies the standard existence and uniqueness of solutions properties for all initial states $x_{0} \in \mathscr{G}$ and all disturbances $\delta \in \mathscr{M}_{\mathscr{U}}$ [6], and $\mathscr{F}(\mathscr{E}, 0)=0$. Let $\mathscr{S} \subseteq \mathscr{G}$ be any neighborhood of $\mathscr{E}$. We say that the system is integral inputto-state stable (iISS) with respect to $(\mathscr{U}, \mathscr{E}, \mathscr{S})$ provided there are functions $\beta \in \mathscr{K} \mathscr{L}$ and $\gamma_{i} \in \mathscr{K}_{\infty}$ and a modulus $\Lambda$ with respect to $\mathscr{S}$ such that $\gamma_{1}\left(\left|x\left(t, x_{0}, \delta\right)\right|_{\mathscr{E}}\right) \leq \beta\left(\Lambda\left(x_{0}\right), t\right)+$ $\int_{0}^{t} \gamma_{2}(|\delta(r)|) \mathrm{d} r$ for all $t \geq 0$, all solutions $x\left(t, x_{0}, \delta\right)$ of the system with initial states $x_{0} \in \mathscr{S}$, and all $\delta \in \mathscr{M}_{\mathscr{U}}$. This agrees with the usual iISS condition from [1], [13] when $\mathscr{G}=\mathscr{S}=\mathbb{R}^{n}$ and $\mathscr{E}=0$. The special case of iISS where $\mathscr{F}$ only depends on $x$ and the integral term in the iISS estimate is not present is global asymptotic stability (GAS) with respect to $(\mathscr{E}, \mathscr{S})$. In that case, a nonstrict (resp., strict) Lyapunov function for the system with respect to $(\mathscr{E}, \mathscr{G})$ is any modulus $\mathscr{V}$ with respect to $(\mathscr{E}, \mathscr{G})$ that is $C^{1}$ on some open set containing $\mathscr{G}$, and that is such that the function $\dot{\mathscr{V}}: \mathscr{G} \rightarrow \mathbb{R}$ defined by $\dot{\mathscr{V}}(q)=\nabla \mathscr{V}(q) \mathscr{F}(q)$ is negative semidefinite (resp., definite) with respect to $\mathscr{E}$. A set $\mathscr{S} \subseteq \mathscr{G}$ is robustly forwardly invariant for the system with disturbances in $\mathscr{U}$ provided all trajectories of the system starting in $\mathscr{S}$ with disturbances $\delta \in \mathscr{M}_{\mathscr{U}}$ remain in $\mathscr{S}$ for all positive times.

\section{PROBlem Formulation}

We can show from physics principles that the curve tracking dynamics of marine robots on the surface of water can be simplified to the equations [17]

$$
\left\{\begin{array}{l}
\dot{\rho}=-\sin (\phi) \\
\dot{\phi}=\frac{\kappa \cos (\phi)}{1+\kappa \rho}-K_{2}\left[u_{2}+\delta\right]
\end{array}\right.
$$

where $\rho$ is the relative distance, $\phi$ is the bearing, $\kappa$ is the (positive) curvature at the closest point, $u_{2}$ is the steering control, the control gain $K_{2}>0$ is constant (and possibly uncertain), the perturbation $\delta \in \mathscr{M}_{\mathbb{R}}$ represents controller uncertainty, and the state space is $\mathscr{X}=(0,+\infty) \times(-\pi / 2, \pi / 2)$. While (1) is feedback linearizable using the new state variable $z=\sin (\phi)$, the feedback linearized system does not respect the state space and so cannot be used [7].

Assuming that $K_{2}$ is known, the work [17] designed a feedback control law to achieve asymptotic stabilization of an equilibrium state corresponding to constant distance $\left(\rho=\rho_{0}>0\right)$ and zero bearing $(\phi=0)$, which occurs when the robot moves parallel to the curve. We call this case the nonadaptive case.

However, $K_{2}$ depends on the vehicle design and the environment, and therefore is unknown in most applications.
In this paper, our goals for an adaptive control law are to have $K_{2}$ identified in addition to achieving $\rho \rightarrow \rho_{0}$ and $\phi \rightarrow 0$ as $t \rightarrow \infty$ from all initial states, when $\delta=0$. Then, we would like to establish iISS properties for the closed loop dynamics with respect to $\delta$ under the adaptive control law. This is more challenging than what has been addressed in our previous works [7], [17] because $K_{2}$ is unknown, and because we must simultaneously show integral ISS properties with respect to $\delta$ and identify $K_{2}$.

We solve this adaptive stabilization and parameter identification problem using a variant of the barrier nonstrict Lyapunov function and 'strictification' methods from [9]. The methods from [9] include parameter identification and integral ISS results with respect to disturbances. However, [9] cannot be applied to (1) directly, since we must ensure that its trajectories $(\rho(t), \phi(t))$ remain in $\mathscr{X}$. Therefore, we provide a variant of the invariant hexagon argument from [7] that ensures robust forward invariance of suitable hexagons for the adaptive dynamics, under maximum bounds on the perturbation $\delta$. This allows us to use the barrier Lyapunov function and strictification approach from [9] to get the stabilization, parameter identification, and integral ISS, which would not be possible using earlier methods.

\section{REVIEW OF NONADAPTIVE CASE}

We next review the controller design from [17] and the ISS and strict Lyapunov function results from [7]. The controllers in [7], [17] have the form

$$
u_{2}=\frac{\kappa \cos (\phi)}{1+\kappa \rho}-h^{\prime}(\rho) \cos (\phi)+\mu \sin (\phi)
$$

where $\mu>0$ is a steering constant. In [7], $h$ is any function that satisfies the following two assumptions:

Assumption 1: The function $h:(0,+\infty) \rightarrow[0,+\infty)$ is $C^{2}, h^{\prime}$ has only finitely many zeros, $\lim _{\rho \rightarrow 0^{+}} h(\rho)=$ $\lim _{\rho \rightarrow+\infty} h(\rho)=+\infty$, and there is a constant $\rho_{0}>0$ such that $h\left(\rho_{0}\right)=0$.

Assumption 2: The function $h$ satisfies Assumption 1 and:

(i) There is an increasing $C^{1}$ function $\gamma:[0,+\infty) \rightarrow[\mu,+\infty)$ such that $\gamma(h(\rho)) \geq \max \left\{\mu, 1+0.5 \mu^{2}+h^{\prime \prime}(\rho)\right\}$ for all $\rho>0$.

(ii) There is a function $\Gamma \in \mathscr{K}_{\infty} \cap C^{1}$ such that $\Gamma(h(\rho)) \geq$ $\left[h^{\prime}(\rho)\right]^{2}$ for all $\rho>0$.

(iii) The function $h^{\prime}(\rho)\left(\rho-\rho_{0}\right)$ is positive for all $\rho>0$ with $\rho \neq \rho_{0}$.

Here $\rho_{0}>0$ is from Assumption 1.

Remark 1: The arguments in [7] show that Assumptions 1-2 hold for

$$
h(\rho)=\alpha\left\{\rho+\frac{\rho_{0}^{2}}{\rho}-2 \rho_{0}\right\}
$$

from [17] for any constants $\alpha>0$ and $\rho_{0}>0$, if we take $\gamma(q)=2\left(q+2 \alpha \rho_{0}\right)^{3} /\left\{\alpha^{2} \rho_{0}^{4}\right\}+1+0.5 \mu^{2}+\mu$ and $\Gamma(q)=$ $\frac{18 \alpha}{\rho_{0}} q+\left(\frac{2}{\rho_{0}}\right)^{4}\left(\frac{9}{\alpha^{2}}\right) q^{4}$.

The works [7], [17] assume that $K_{2}=1$, in which case substituting (2) into (1) produces the closed loop system

$$
\left\{\begin{aligned}
\dot{\rho} & =-\sin (\phi) \\
\dot{\phi} & =h^{\prime}(\rho) \cos (\phi)-\mu \sin (\phi)
\end{aligned}\right.
$$


on $\mathscr{X}=(0,+\infty) \times(-\pi / 2, \pi / 2)$ when no perturbation $\delta$ is present. Under Assumption 1, [17] shows GAS of (4) with respect to $\left(\left(\rho_{0}, 0\right), \mathscr{X}\right)$, using the nonstrict Lyapunov function

$$
V(\rho, \phi)=-\ln (\cos (\phi))+h(\rho)
$$

for (4) with respect to $\left(\left(\rho_{0}, 0\right), \mathscr{X}\right)$ and LaSalle Invariance. For robustness, the arguments from [7] show that (4) admits the strict Lyapunov function

$$
\begin{aligned}
U_{2}(\rho, \phi)= & -h^{\prime}(\rho) \sin (\phi)+\frac{1}{\mu} \int_{0}^{V(\rho, \phi)} \gamma(m) \mathrm{d} m \\
& +\Gamma(V(\rho, \phi))+V(\rho, \phi)
\end{aligned}
$$

with respect to $\left(\left(\rho_{0}, 0\right), \mathscr{X}\right)$ when $V$ is given by (5). In fact, along all trajectories of (4) in $\mathscr{X}$, we have $U_{2} \geq V$ and

$$
\begin{aligned}
\dot{U}_{2} \leq & -0.5\left[h^{\prime}(\rho) \cos (\phi)\right]^{2}-\sin ^{2}(\phi) \\
& -\left[\Gamma^{\prime}(V(\rho, \phi))+1\right] \mu \frac{\sin ^{2}(\phi)}{\cos (\phi)},
\end{aligned}
$$

whose right side is negative definite with respect to $\left(\rho_{0}, 0\right)$. This allowed us to prove ISS results with respect to additive uncertainty on the controller, under certain restrictions on the magnitude of $\delta$ that maintain forward invariance properties.

\section{Adaptive Controller Design}

We aim to extend [7] to the constant speed adaptive curve tracking dynamics (1) where $K_{2}$ is to be identified (but see Remark 3 below for a further extension involving adaptive speed control with unknown control gains). In practice, positive lower and upper bounds for $K_{2}$ are known. Therefore, we assume the following in the rest of this paper:

Assumption 3: There are known constants $c_{\min }>0$ and $c_{\max }>0$ such that $c_{\min }<K_{2}<c_{\max }$.

Following [9], we take the update law

$$
\dot{\widehat{K_{2}}}=\left(\widehat{K}_{2}-c_{\min }\right)\left(c_{\max }-\widehat{K}_{2}\right) \mho
$$

where the estimator $\widehat{K}_{2}$ for the parameter $K_{2}$ has the state space $\left(c_{\min }, c_{\max }\right)$ and the locally Lipschitz function $\mho$ is to be specified. ${ }^{1}$ Denoting the adaptive controller by $v$ gives

$$
\left\{\begin{aligned}
\dot{\rho} & =-\sin (\phi) \\
\dot{\phi} & =\frac{\kappa \cos (\phi)}{1+\kappa \rho}-K_{2} v-K_{2} \delta \\
\dot{\widehat{K}}_{2} & =\left(\widehat{K}_{2}-c_{\min }\right)\left(c_{\max }-\widehat{K}_{2}\right) \mho
\end{aligned}\right.
$$

with state variables $\left(\rho, \phi, \widehat{K}_{2}\right)$. Choosing $v=u_{2} / \widehat{K}_{2}$ and $\mho=$ $-\left(\partial U_{2} / \partial \phi\right) v$, where $u_{2}$ is the controller from (2) and $U_{2}$ is

\footnotetext{
${ }^{1}$ A simple uniqueness of solutions argument shows that each trajectory of (8) starting in $\left(c_{\min }, c_{\max }\right)$ remains in $\left(c_{\min }, c_{\max }\right)$ at all positive times. To see why, suppose that $K_{*}(t)$ were a solution of (8) starting in $\left(c_{\min }, c_{\max }\right)$ that reached $c_{\max }$ at some positive time $\bar{t}$. Then the time reversed system would admit two distinct solutions for the initial state $c_{\max }$ on $[0, \bar{t}]$, namely, the constant solution at $c_{\max }$ and $t \mapsto K_{*}(\bar{t}-t)$, contradicting the uniqueness property for solutions. Similar reasoning applies to the other endpoint $c_{\min }$.
}

the strict Lyapunov function from (6), gives the augmented tracking and parameter estimation dynamics

$$
\left\{\begin{aligned}
\dot{\rho}= & -\sin (\phi) \\
\dot{\phi}= & \left(1-\frac{K_{2}}{\widehat{K}_{2}}\right) \frac{\kappa \cos (\phi)}{1+\kappa \rho} \\
& +\frac{K_{2}}{\widehat{K}_{2}}\left[h^{\prime}(\rho) \cos (\phi)-\mu \sin (\phi)\right]-K_{2} \delta \\
\dot{\widehat{K}}_{2}= & -\left(\widehat{K}_{2}-c_{\min }\right)\left(c_{\max }-\widehat{K}_{2}\right) \frac{\partial U_{2}}{\partial \phi} \frac{u_{2}}{\widehat{K}_{2}} .
\end{aligned}\right.
$$

We wish to establish GAS and iISS properties for (10). However, the desired state space $\mathscr{X}^{b}=(0,+\infty) \times(-\pi / 2, \pi / 2) \times$ $\left(c_{\min }, c_{\max }\right)$ will not be forward invariant for (10) unless we impose restrictions on the magnitude of $\delta$. To see why, choose the initial states $(\rho(0), \phi(0))=\left(2 \rho_{0}, 0\right)$, any initial value for $\widehat{K}_{2}$ in $\left[c_{\min }, c_{\max }\right]$, and the constant perturbation

$$
\delta=-\frac{1}{c_{\min }}\left[\frac{c_{\max }}{c_{\min }}(\kappa+\mu)+\frac{\pi}{2 \rho_{0}}\right],
$$

and suppose that $(\rho(t), \phi(t), \widehat{K}(t))$ were the corresponding trajectory for (10) defined on $\left[0, \rho_{0}\right]$. Then $\rho(t) \geq \rho_{0}$ for all $t \in\left[0, \rho_{0}\right]$, so $h^{\prime}(\rho(t)) \geq 0$ for all $t \in\left[0, \rho_{0}\right]$. Hence, $\dot{\phi}(t) \geq$ $-\frac{c_{\max }}{c_{\min }}(\kappa+\mu)-K_{2} \delta \geq \frac{\pi}{2 \rho_{0}}$ for all $t \in\left[0, \rho_{0}\right]$, which would give $\phi\left(\rho_{0}\right) \geq \pi / 2$. We turn to our restrictions on $\delta$ next.

\section{FORWARDLY INVARIANT HEXAGONS}

We extend the forwardly invariant hexagon arguments from [7] to the adaptive dynamics (10), which will lead to iISS results for (10) under suitable restrictions on the magnitude of $\delta$. Since there is no disturbance in the $\widehat{K}_{2}$ dynamics in (10), maintaining forward invariance of $\mathscr{X}^{b}$ only involves the $(\rho, \phi)$ subdynamics

$$
\left\{\begin{aligned}
\dot{\rho}= & -\sin (\phi) \\
\dot{\phi}= & \cos (\phi)\left[\frac{\kappa}{1+\kappa \rho}\left(1-\frac{K_{2}}{\widehat{K}_{2}}\right)+\frac{K_{2}}{\widehat{K}_{2}} h^{\prime}(\rho)\right] \\
& -\frac{K_{2}}{\widehat{K}_{2}} \mu \sin (\phi)-K_{2} \delta
\end{aligned}\right.
$$

of (10), where $K_{2}$ and its estimator $\widehat{K}_{2}$ both lie on $\left(c_{\min }, c_{\max }\right)$ as before. We now add the assumption that the function $h$ satisfying Assumptions 1-2 also satisfies:

Assumption 4: The function $h$ satisfies $\lim _{\rho \rightarrow 0^{+}} h^{\prime}(\rho)=$ $-\infty$ and $\liminf _{\rho \rightarrow \infty} h^{\prime}(\rho)>0$. Also, $h^{\prime \prime}\left(\rho_{0}\right)>0$.

Notice that Assumption 4 holds for the choice (3) of $h$ for any choices of its constants $\alpha>0$ and $\rho_{0}>0$. Using Assumption 4, we can choose a sufficiently small constant $\rho_{*}>0$ and a sufficiently large constant $\bar{K}>0$ such that

$$
\rho_{*} \in\left(0, \frac{\rho_{0}}{1+c_{\max } / c_{\min }}\right) \text { and } \bar{K}>1+\frac{c_{\max }}{c_{\min }}
$$

hold, and such that

$$
\min \left\{\left|h^{\prime}(\rho)\right|: \rho_{*} \leq \rho \leq B_{1}\right\}>\kappa\left(\frac{c_{\max }}{c_{\min }}\right)^{2}
$$

and

$$
\min \left\{h^{\prime}(\rho): \bar{K} \rho_{0} \leq \rho \leq D_{1}\right\}>\frac{\kappa}{1+\kappa \bar{K} \rho_{0}}\left(\frac{c_{\max }}{c_{\min }}\right)^{2}
$$

where $B_{1}=\rho_{*}\left(1+c_{\max } / c_{\min }\right)$ and $D_{1}=\bar{K} \rho_{0}+\rho_{*} c_{\max } / c_{\min }$. (To see why we can satisfy (13)-(15), let $I_{0}$ denote the liminf from Assumption 4. The left side of (14) goes to $+\infty$ as 


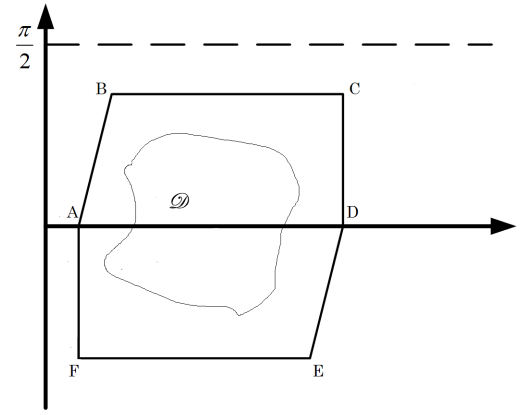

Fig. 1. Forwardly Invariant Hexagon $\widetilde{H}\left(\rho_{*}, \mu, \bar{K}\right)$ in $(\rho, \phi)$ Plane

$\rho_{*} \rightarrow 0^{+}$so we can satisfy (14) for small enough $\rho_{*}>0$, while the left side of (15) is above $I_{0} / 2$ if $\bar{K}$ is large enough. Then we can satisfy (15) by taking $\bar{K}$ big enough to make the right side of (15) less than $I_{0} / 2$.) Our condition on $\bar{K}$ ensures that $\bar{K} \rho_{0}>B_{1}$. Pick any constant $\mu \in\left(0, \pi /\left(2 \rho_{*}\right)\right)$ such that

$$
\mu \tan \left(\mu \rho_{*}\right)>\left(c_{\max } / c_{\min }\right)^{2}\left[\kappa+\left|h^{\prime}\right|_{\left[\rho_{*}, D_{1}\right]}\right],
$$

by choosing $\mu$ close enough to but below $\pi /\left(2 \rho_{*}\right)$.

Let $\widetilde{H}\left(\rho_{*}, \mu, \bar{K}\right)$ denote the region in the $(\rho, \phi)$ plane that is bounded by the hexagon having vertices $A=\left(\rho_{*}, 0\right), B=$ $\left(B_{1}, \mu \rho_{*}\right), C=\left(D_{1}, \mu \rho_{*}\right), D=\left(D_{1}, 0\right), E=\left(\bar{K} \rho_{0},-\mu \rho_{*}\right)$, and $F=\left(\rho_{*},-\mu \rho_{*}\right)$. Its legs $A B$ and $E D$ have the slope $\mu^{\sharp}=c_{\min } \mu / c_{\max }$. These hexagons agree with the ones for the nonadaptive case in [7], except that the tilted legs in [7] have slope $\mu$. For each compact subset $\mathscr{D}$ of $\mathscr{X}=(0,+\infty) \times$ $(-\pi / 2, \pi / 2)$, we can choose $\rho_{*}, \bar{K}$, and $\mu$ to satisfy (13)(16) and to be such that $\mathscr{D} \subseteq \widetilde{H}\left(\rho_{*}, \mu, \bar{K}\right)$. This can be done by moving $\rho_{*}>0$ far enough to the left and $\bar{K} \rho_{0}$ far enough to the right, and making $\mu$ large enough and close enough to $\pi /\left(2 \rho_{*}\right)$ so that the hexagon approximates a rectangle whose top and bottom are as close as desired to $\pm \pi / 2$. See Fig. 1 . Given any compact subset $\mathscr{D} \subseteq \mathscr{X}$, our work will establish robust forward invariance of any hexagon that contains $\mathscr{D}$.

To simplify the analysis, we set

$$
\begin{aligned}
& Q_{1}\left(\rho, \phi, \widehat{K}_{2}, K_{2}\right)= \\
& \cos (\phi)\left(\frac{\kappa}{1+\kappa \rho} \frac{\widehat{K}_{2}-K_{2}}{\widehat{K}_{2}}+\frac{K_{2}}{\widehat{K}_{2}} h^{\prime}(\rho)-\frac{K_{2}}{\widehat{K}_{2}} \mu \tan (\phi)\right) \text { and } \\
& Q_{2}\left(\rho, \phi, \widehat{K}_{2}, K_{2}\right)=Q_{1}\left(\rho, \phi, \widehat{K}_{2}, K_{2}\right)+\mu^{\sharp} \sin (\phi) .
\end{aligned}
$$

We use the following lemma [8]:

Lemma 1: For all constants $K_{2} \in\left[c_{\min }, c_{\max }\right]$ and $\widehat{K}_{2} \in$ $\left[c_{\min }, c_{\max }\right]$, we have: (a) $Q_{1}\left(\rho, \phi, \widehat{K}_{2}, K_{2}\right)<0$ (resp., $>0$ ) for all $(\rho, \phi) \in B C$ (resp., $F E$ ). (b) $Q_{2}\left(\rho, \phi, \widehat{K}_{2}, K_{2}\right)<0$ (resp., $>0$ ) for all $(\rho, \phi) \in A B$ (resp., $E D)$.

By Lemma 1, the following constants are positive:

$$
\begin{aligned}
& \Delta_{*}= \\
& \min \left\{\left|Q_{1}\left(\rho, \phi, \widehat{K}_{2}, K_{2}\right)\right| / K_{2}:\right. \\
& \left.(\rho, \phi) \in B C \cup E F, K_{2} \in\left[c_{\min }, c_{\max }\right], \widehat{K}_{2} \in\left[c_{\min }, c_{\max }\right]\right\} \\
& \Delta_{* *}= \\
& \min \left\{\left|Q_{2}\left(\rho, \phi, \widehat{K}_{2}, K_{2}\right)\right| / K_{2}:\right. \\
& \left.(\rho, \phi) \in A B \cup E D, K_{2} \in\left[c_{\min }, c_{\max }\right], \widehat{K}_{2} \in\left[c_{\min }, c_{\max }\right]\right\}
\end{aligned}
$$

Fix any constant $\delta_{*} \in\left(0, \min \left\{\Delta_{*}, \Delta_{* *}\right\}\right)$. We prove the following robust forward invariance result for (12):

Theorem 1: Let Assumptions 1-4 hold. Let $\rho_{*}$ and $\bar{K}$ be any constants satisfying (13)-(15). Choose any constant $\mu \in$ $\left(0, \pi /\left(2 \rho_{*}\right)\right)$ satisfying (16), any constant $K_{2} \in\left[c_{\min }, c_{\max }\right]$, any function $\widehat{K}_{2}:[0, \infty) \rightarrow\left[c_{\min }, c_{\max }\right]$, and any measurable function $\delta:[0, \infty) \rightarrow\left[-\delta_{*}, \delta_{*}\right]$. Then all trajectories of (12) starting in $\widetilde{H}\left(\rho_{*}, \mu, \bar{K}\right)$ stay in $\widetilde{H}\left(\rho_{*}, \mu, \bar{K}\right)$ for all $t \geq 0$.

Proof: We prove that for each such trajectory $(\rho, \phi)(t)$ of (12) starting in $\widetilde{H}\left(\rho_{*}, \mu, \bar{K}\right)$, the vector field $(\dot{\rho}, \dot{\phi})$ points into the hexagon along all six legs of the hexagon, so the trajectory cannot leave the hexagon. By part (a) of Lemma 1, we have $\dot{\phi}=Q_{1}-K_{2} \delta \leq Q_{1}+K_{2} \delta_{*}<0$ on $B C$ because $\delta_{*}<$ $\Delta_{*}$. Along $F E$, part (a) of Lemma 1 gives $\dot{\phi}=Q_{1}-K_{2} \delta \geq$ $Q_{1}-K_{2} \delta_{*}>0$ along $F E$, again because $\delta_{*}<\Delta_{*}$. Hence, the trajectories point down (resp., up) along the top (resp., bottom) leg. Along $A F$, we have $\dot{\rho}>0$, except at the point $A$. At $A$, we have $\dot{\rho}=0$ and $\dot{\phi}<0$, because $\delta_{*}<\Delta_{* *}$, which makes $\rho$ increase. Along the leg $C D$, we have $\dot{\rho}<0$, except at $D$. At $D$, we have $\dot{\rho}=0$ and $\dot{\phi}>0$, because $\delta_{*}<\Delta_{* *}$, which makes $\rho$ decrease. Hence we cannot exit the hexagon though its top, bottom, or vertical legs.

To rule out exits through the tilted legs, we use $\mathscr{I}(\rho, \phi)=$ $\phi-\mu^{\sharp} \rho$. For each pair $(\rho, \phi) \in \mathscr{X}$, the value $\mathscr{I}(\rho, \phi)$ is the $\phi$-axis intercept of the line through $(\rho, \phi)$ having slope $\mu^{\sharp}$, so the theorem will follow once we show that $\dot{\mathscr{I}}<0$ (resp., $\dot{\mathscr{I}}>0$ ) along $A B$ (resp., $E D)$. Since $\delta_{*}<\Delta_{* *}$, it follows from part (b) of Lemma 1 that along $A B$, we have $\dot{\mathscr{I}}=$ $Q_{2}-K_{2} \delta<0$. This rules out exits along $A B$. Again using part (b) of Lemma 1 and the fact that $\delta_{*}<\Delta_{* *}$, we also have $\dot{\mathscr{I}}=Q_{2}-K_{2} \delta>0$ for all $(\rho, \phi) \in D E$. This rules out exits along $D E$, completing the proof.

Remark 2: Given any function $h$ satisfying Assumptions 1,2 , and 4 , the bound $\min \left\{\Delta_{*}, \Delta_{* *}\right\}$ is the supremum of all possible perturbation bounds that render $\widetilde{H}\left(\rho_{*}, \mu, \bar{K}\right)$ robustly forward invariant for (12) for all $K_{2}$ and all signals $\widehat{K}_{2}(t)$, and therefore is optimal. In fact, for each constant $\tilde{\delta}>$ $\min \left\{\Delta_{*}, \Delta_{* *}\right\}$, we can find a point $\widetilde{p}$ on the boundary of $\widetilde{H}\left(\rho_{*}, \mu, \bar{K}\right)$ such that the trajectory of (12) starting at $\widetilde{p}$ for one of the constant perturbations $\pm \widetilde{\delta}$ and some admissible choices of $K_{2}$ and $\widehat{K}_{2}$ exits the hexagon. To see why, note that if $\min \left\{\Delta_{*}, \Delta_{* *}\right\}=\Delta_{* *}$ and if $\tilde{\delta}>\Delta_{* *}=-Q_{2}\left(\rho, \phi, \widehat{K}_{2}, K_{2}\right) / K_{2}$ at some $\widetilde{p}=(\rho, \phi)^{\top} \in \mathrm{AB}$, then taking the constant disturbance $\delta=-\tilde{\delta}$ gives $\dot{\mathscr{I}}=Q_{2}\left(\rho, \phi, \widehat{K}_{2}, K_{2}\right)+K_{2} \tilde{\delta}>0$ at $\tilde{p}$, so we exit from $\tilde{p}$. On the other hand, if $\tilde{\delta}>\Delta_{* *}=$ $Q_{2}\left(\rho, \phi, \widehat{K}_{2}, K_{2}\right) / K_{2}$ at some $\tilde{p}=(\rho, \phi)^{\top} \in \mathrm{ED}$, then taking the constant disturbance $\delta=\tilde{\delta}$ gives $\dot{\mathscr{I}}=Q_{2}\left(\rho, \phi, \widehat{K}_{2}, K_{2}\right)-$ $K_{2} \tilde{\delta}<0$, so we again exit at $\widetilde{p}$. The other cases where $\min \left\{\Delta_{*}, \Delta_{* *}\right\}=\Delta_{*}$ are handled similarly. Hence, for each choice of $h$, our bound $\delta_{*}$ is optimal for maintaining the necessary invariance properties of $\widetilde{H}\left(\rho_{*}, \mu, \bar{K}\right)$ for (12) and not at all conservative, and our controller is independent of the choice of the hexagon.

\section{InTEGRAL InPUT-TO-STATE STABILITY}

The preceding section shows that for each hexagon $\widetilde{H}\left(\rho_{*}, \mu, \bar{K}\right)$ and each bound $\delta_{*}$ on the admissible actuator 
error $\delta$ satisfying the requirements from Theorem 1, the augmented tracking and parameter estimation dynamics

$$
\left\{\begin{array}{l}
\dot{\tilde{q}}_{1}=-\sin \left(\widetilde{q}_{2}\right) \\
\dot{\widetilde{q}}_{2}=\frac{\kappa \cos \left(\widetilde{q}_{2}\right)}{1+\kappa\left(\widetilde{q}_{1}+\rho_{0}\right)}-\frac{K_{2}}{\widehat{K}_{2}} u_{2}-K_{2} \delta \\
\dot{\widehat{K}}_{2}=-\left(\widehat{K}_{2}-c_{\min }\right)\left(c_{\max }-\widehat{K}_{2}\right) \frac{\partial U_{2}}{\partial \phi} \frac{u_{2}}{\widehat{K}_{2}}
\end{array}\right.
$$

with state variables $\widetilde{q}=\left(\widetilde{q}_{1}, \widetilde{q}_{2}\right)=\left(\rho-\rho_{0}, \phi\right)$ and $\widehat{K}_{2}$ and disturbances $\delta$ valued in $\mathscr{U}=\left[-\delta_{*}, \delta_{*}\right]$ has the robustly forward invariant set

$$
\mathscr{X}^{+}=\left(\widetilde{H}\left(\rho_{*}, \mu, \bar{K}\right)-\mathscr{E}\right) \times\left(c_{\min }, c_{\max }\right) .
$$

Here $u_{2}$ is the controller from (2), $U_{2}$ is the strict Lyapunov function from (6), and we subtract the equilibrium $\mathscr{E}=$ $\left(\rho_{0}, 0\right)$ in $(21)$ to account for going from the original curve tracking dynamics for $q=(\rho, \phi)$ to the error dynamics for $\widetilde{q}$. It is therefore meaningful to ask whether the dynamics

$$
\left\{\begin{array}{l}
\dot{\widetilde{q}}_{1}=-\sin \left(\widetilde{q}_{2}\right) \\
\dot{\widetilde{q}}_{2}=\frac{\kappa \cos \left(\widetilde{q}_{2}\right)}{1+\kappa\left(\widetilde{q}_{1}+\rho_{0}\right)}-u_{2}+\frac{\widetilde{K}_{2}}{\widetilde{K}_{2}+K_{2}} u_{2}-K_{2} \delta \\
\dot{\widetilde{K}}_{2}=-\left(\widetilde{K}_{2}+K_{2}-c_{\min }\right)\left(c_{\max }-\widetilde{K}_{2}-K_{2}\right) \frac{\partial U_{2}}{\partial \phi} \frac{u_{2}}{\widetilde{K}_{2}+K_{2}}
\end{array}\right.
$$

for $\left(\widetilde{q}, \widetilde{K}_{2}\right)=\left(\rho-\rho_{0}, \phi, \widehat{K}_{2}-K_{2}\right)$ is iISS with respect to $\left(\mathscr{U}, 0, \mathscr{X}^{\sharp}\right)$ with the choices $\mathscr{U}=\left[-\delta_{*}, \delta_{*}\right]$ and

$$
\mathscr{X}^{\sharp}=\left(\widetilde{H}\left(\rho_{*}, \mu, \bar{K}\right)-\mathscr{E}\right) \times\left(c_{\min }-K_{2}, c_{\max }-K_{2}\right),
$$

since this would give stabilization of the equilibrium point $\left(\rho_{0}, 0\right)$ and parameter identification when $\delta=0$, as well as robustness in the sense of iISS on each hexagon. This is much stronger than local stabilization, because the hexagons can be arbitrarily wide. In [8], we use ideas from [9] to prove that the iISS property is indeed satisfied. Here we summarize the ideas behind our iISS result. Throughout this section, we fix a hexagon $\widetilde{H}\left(\rho_{*}, \mu, \bar{K}\right)$ and a corresponding disturbance bound $\delta_{*}$ as in Theorem 1, so all disturbances $\delta$ are valued in $\mathscr{U}=\left[-\delta_{*}, \delta_{*}\right]$. To simplify the notation, we sometimes denote the hexagon simply by $\widetilde{H}$.

Following [9], we first take the barrier nonstrict Lyapunov function $U_{3}: \mathscr{X}^{\sharp} \rightarrow[0,+\infty)$ defined by

$$
\begin{aligned}
& U_{3}\left(\widetilde{q}, \widetilde{K}_{2}\right)= \\
& U_{2}\left(\widetilde{q}+\left(\rho_{0}, 0\right)\right)+\int_{0}^{\widetilde{K}_{2}} \frac{\ell}{\left(\ell+K_{2}-c_{\min }\right)\left(c_{\max }-\ell-K_{2}\right)} \mathrm{d} \ell .
\end{aligned}
$$

A partial fraction decomposition shows that the integral in (24) can be rewritten as

$$
\frac{c_{\min }-K_{2}}{c_{\max }-c_{\min }} \ln \left|\frac{\widetilde{K}_{2}+K_{2}-c_{\min }}{K_{2}-c_{\min }}\right|-\frac{c_{\max }-K_{2}}{c_{\max }-c_{\min }} \ln \left|\frac{c_{\max }-\widetilde{K}_{2}-K_{2}}{c_{\max }-K_{2}}\right|
$$

and so is a modulus with respect to $(0, \mathscr{W})$ where $\mathscr{W}=$ $\left(c_{\min }-K_{2}, c_{\max }-K_{2}\right)$. Moreover, the decay estimate (7) along all trajectories of (4) gives

$$
\begin{aligned}
\dot{U}_{3} \leq & -0.5\left[h^{\prime}(\rho) \cos (\phi)\right]^{2}-\sin ^{2}(\phi) \\
& -\left[\Gamma^{\prime}(V(\rho, \phi))+1\right] \mu \frac{\sin ^{2}(\phi)}{\cos (\phi)}-K_{2} \frac{\partial U_{2}}{\partial \phi}(\rho, \phi) \delta
\end{aligned}
$$

along all trajectories of (22). Since $K_{2}\left(\partial U_{2} / \partial \phi\right)$ is bounded on $\mathscr{X}^{\sharp}$, condition (25) resembles the iISS Lyapunov function decay condition [1], save for the fact that its right side is not negative definite in the full state $\left(\widetilde{q}, \widetilde{K}_{2}\right)$ when $\delta=0$. Also, the adaptive arguments from [9] do not apply directly because of the restricted state space and the actuator error. On the other hand, we can use $U_{3}$ to prove [8]:

Theorem 2: Let $h, \rho_{*}, \mu, \bar{K}$, and $\delta_{*}$ satisfy Assumptions 1-2 and the requirements of Section VI. Then (22) is iISS with respect to $\left(\left[-\delta_{*}, \delta_{*}\right], 0, \mathscr{X}^{\sharp}\right)$.

Remark 3: The preceding analysis assumed that the speed of the robot is constant. However, we can extend it to include adaptive speed control. For details, see [8].

\section{Simulations}

To illustrate our stabilization, parameter identification, and iISS results, we simulated (22) with the choice

$$
h(\rho)=10\left(\rho+\frac{1}{\rho}-2\right) .
$$

This satisfies our assumptions with $\alpha=10$ and $\rho_{0}=1$; see Remark 1. We used the functions $\gamma$ and $\Gamma$ from Remark 1 to generate the adaptive controls. We also took $c_{\min }=0.5$, $c_{\max }=0.75, \kappa=2, \rho_{*}=0.12, \mu=0.995 \pi /\left(2 \rho_{*}\right)=13.025$ and $\bar{K}=10$, which give the hexagon $\widetilde{H}(0.12,13.025,10) \subseteq$ $\mathscr{X}$. These parameter values satisfy our requirements (13)(16). Using the notation from above, our assumptions are satisfied with $\delta_{*}=0.103671$. In our first simulation, we took the initial values $\left(\widetilde{q}, \widetilde{K}_{2}, \widehat{K}_{2}\right)(0)=((0.2,0.4), 0.2,0.7)$ and the disturbance $\delta \equiv 0$ and obtained the trajectory components for $\left(\widetilde{q}, \widetilde{K}_{2}\right)(t)$ in Figure 2, which shows rapid convergence of the state and parameter estimation errors to zero. Our second simulation was the same as our first, except we included the sinusoidal disturbance $\delta(t)=0.1 \sin (t)$. In Figure 3, we show the simulated trajectory for $\widetilde{q}(t)$ for the second simulation. We do not show the $\widetilde{K}_{2}$ trajectory for our second simulation with the disturbance, because it was the same as for the undisturbed case. With the nonzero disturbance, the errors $\widetilde{q}(t)$ no longer converge to zero. Instead, there is a small overshoot in $\widetilde{q}(t)$, which is made precise by the corresponding overshoot in the iISS estimate. This illustrates the effects of introducing a disturbance in the adaptive stabilization and parameter estimation dynamics.

\section{COnClusions}

Adaptive stabilization and parameter identification for robotic curve tracking models with unknown control gains under controller uncertainty is a challenging problem that is beyond the scope of the standard adaptive control methods. This is due to the restricted state space, the need for strict Lyapunov functions for the augmented stabilization and estimation dynamics, and the need to identify the parameters instead of merely ensuring that the parameter estimate stays bounded. We used recently developed barrier Lyapunov function and strictification approaches to solve the stabilization and parameter identification problems and prove robustness under controller uncertainty in the sense of integral input-tostate stability. We aim to extend our work to curve tracking problems under time delays, and to three-dimensional curve tracking. 

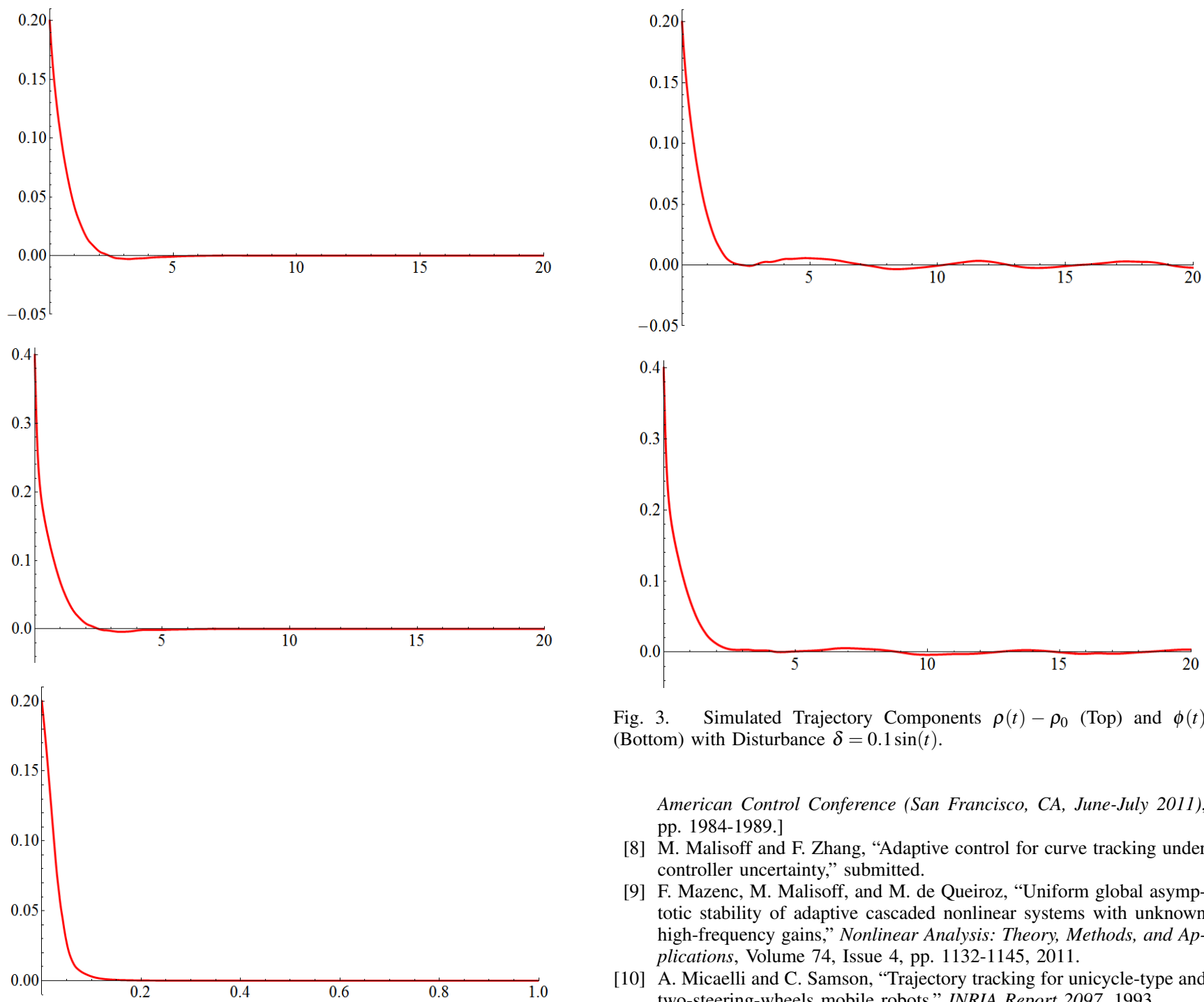

Fig. 3. Simulated Trajectory Components $\rho(t)-\rho_{0}$ (Top) and $\phi(t)$ (Bottom) with Disturbance $\delta=0.1 \sin (t)$.

American Control Conference (San Francisco, CA, June-July 2011), pp. 1984-1989.]

[8] M. Malisoff and F. Zhang, "Adaptive control for curve tracking under controller uncertainty," submitted.

[9] F. Mazenc, M. Malisoff, and M. de Queiroz, "Uniform global asymptotic stability of adaptive cascaded nonlinear systems with unknown high-frequency gains," Nonlinear Analysis: Theory, Methods, and Applications, Volume 74, Issue 4, pp. 1132-1145, 2011.

[10] A. Micaelli and C. Samson, "Trajectory tracking for unicycle-type and two-steering-wheels mobile robots," INRIA Report 2097, 1993.

Fig. 2. Simulated Trajectory Components $\rho(t)-\rho_{0}$ (Top), $\phi(t)$ (Middle), and $\widetilde{K}_{2}$ (Bottom) with Disturbance $\delta \equiv 0$.

\section{REFERENCES}

[1] D. Angeli, E.D. Sontag, and Y. Wang, "A characterization of integral input-to-state stability," IEEE Transactions on Automatic Control, Volume 45, Issue 6, pp. 1082-1097, 2000.

[2] http://users.ece.gatech.edu/ fumin/GrandIsle.html

[3] E. Justh and P. Krishnaprasad, "Natural frames and interacting particles in three dimensions," in Proceedings of the 44th IEEE Conference on Decision and Control and European Control Conference (Seville, Spain, December 2005), pp. 2841-2846.

[4] R. Lenain, B. Thuilot, C. Cariou, and P. Martinet, "High accuracy path tracking for vehicles in presence of sliding: Application to farm vehicle automatic guidance for agricultural tasks," Autonomous Robots, Volume 21, Number 1, pp. 79-97, 2006.

[5] V. Lumelsky and A. Stepanov, "Path planning strategies for a point mobile automaton moving amidst unknown obstacles of arbitrary shape," Algorithmica, Volume 2, Number 2, pp. 403-430, 1987.

[6] M. Malisoff and F. Mazenc, Constructions of Strict Lyapunov Functions, Communications and Control Engineering Series, SpringerLondon Ltd., London, UK, 2009.

[7] M. Malisoff, F. Mazenc, and F. Zhang, "Stability and robustness analysis for curve tracking control using input-to-state stability," IEEE Transactions on Automatic Control, to appear. [Summarized Version: M. Malisoff, F. Mazenc, and F. Zhang, "Input-to-state stability for curve tracking control: A constructive approach," in Proceedings of the

[11] P. Morin and C. Samson, "Motion control of wheeled mobile robots," in B. Siciliano and O. Khatib, editors, Springer Handbook of Robotics, pp. 799-826. Springer-Verlag, Berlin, Germany, 2008.

[12] E.D. Sontag, "Stabilizability, i/o stability and coprime factorizations," in Proceedings of the IEEE Conference on Decision and Control (Austin, TX, December 1988), pp. 457-458.

[13] E.D. Sontag, "Comments on integral variants of ISS," Systems and Control Letters, Volume 34, Issues 1-2, pp. 93-100, 1998.

[14] E.D. Sontag, "Input-to-state stability: Basic concepts and results," in P. Nistri and G. Stefani, editors, Nonlinear and Optimal Control Theory, pp. 163-220. Springer, Berlin, Germany, 2008.

[15] C. Woolsey and L. Techy, "Cross-track control of a slender, underactuated AUV using potential shaping," Ocean Engineering: Special Issue on AUVs, Volume 36, Issue 1, pp. 82-91, 2009.

[16] F. Zhang, D.M. Fratantoni, D. Paley, J. Lund, and N. Leonard, "Control of coordinated patterns for ocean sampling," International Journal of Control, Volume 80, Number 7, pp. 1186-1199, 2007.

[17] F. Zhang, E. Justh, and P. Krishnaprasad, "Boundary following using gyroscopic control," in Proceedings of the 43rd IEEE Conference on Decision and Control (Paradise Island, Bahamas, December 2004), pp. 5204-5209.

[18] F. Zhang and N. Leonard, "Coordinated patterns of unit speed particles on a closed curve," Systems and Control Letters, Volume 56, Number 6, pp. 397-407, 2007.

[19] F. Zhang, A. O'Connor, D. Luebke, and P. Krishnaprasad, "Experimental study of curvature-based control laws for obstacle avoidance," in Proceedings of the IEEE International Conference on Robotics and Automation (New Orleans, LA, April-May 2004), pp. 3849-3854. 